ANNALES

POLONICI MATHEMATICI

$87(2005)$

\title{
Whitney triangulations of semialgebraic sets
}

\author{
by Masahiro Shiota (Nagoya)
}

\begin{abstract}
A compact semialgebraic set admits a semialgebraic triangulation such that the family of open simplexes forms a Whitney stratification and is compatible with a finite number of given semialgebraic subsets.
\end{abstract}

A semialgebraic triangulation of a compact semialgebraic set $X$ is a pair of a simplicial complex $K$ and a semialgebraic homeomorphism $f:|K| \rightarrow X$ such that for each $\sigma \in K, f(\operatorname{Int} \sigma)$ is a $C^{\omega}$ manifold and $\left.f\right|_{\operatorname{Int} \sigma}$ is a $C^{\omega}$ diffeomorphism onto $f(\operatorname{Int} \sigma)$, where $|K|$ denotes the underlying polyhedron of $K$. As a consequence of the theory developed in $\left[\mathrm{S}_{2}\right]$ we have:

TheOREM. A compact semialgebraic set $X$ admits a semialgebraic triangulation $(K, f)$ such that $\{f(\operatorname{Int} \sigma): \sigma \in K\}$ is a Whitney stratification of $X$ and the triangulation is compatible with a finite number of given semialgebraic subsets $Y_{i}$ of $X$.

When we work in an o-minimal structure, let the above diffeomorphisms be of class $C^{r}$ for $r \in \mathbb{N}$. Then the arguments below go through. Most of the following maps and manifolds are of class $C^{2}$. However, it is possible to work in the $C^{1}$ category. See $\left[\mathrm{S}_{2}\right]$ for that case.

A (semialgebraic) $C^{2}$ tube about a (semialgebraic) $C^{2}$ manifold $M \subset \mathbb{R}^{n}$ is a triple $T=(|T|, \tau, \varrho)$, where $|T|$ is a (semialgebraic) neighborhood of $M$ in $\mathbb{R}^{n}, \tau:|T| \rightarrow M$ is a (semialgebraic) submersive $C^{2}$ retraction, and $\varrho$ is a nonnegative (semialgebraic) $C^{2}$ function on $|T|$ such that $\varrho^{-1}(0)=M$ and each point $x$ of $M$ is a unique and nondegenerate critical point of the restriction of $\varrho$ to $\tau^{-1}(x)$. The ambient space of $M$ is not necessarily a Euclidean space. It may be a (semialgebraic) $C^{2}$ manifold possibly with boundary. We also define a controlled (semialgebraic) $C^{2}$ tube system for a (semialgebraic) $C^{2}$ stratification in the same way as in the $C^{\infty}$ case (see [G-al] for the $C^{\infty}$ case).

2000 Mathematics Subject Classification: Primary 14P10.

Key words and phrases: semialgebraic sets, Whitney stratifications, triangulations. 
Let $\left\{M_{k}\right\}_{k=1,2, \ldots}$ and $\left\{N_{k}\right\}$ be Whitney $C^{2}$ stratifications of sets $M$ and $N$, respectively, in $\mathbb{R}^{n}$. Let $\left\{T_{k}^{M}=\left(\left|T_{k}^{M}\right|, \tau_{k}^{M}, \varrho_{k}^{M}\right)\right\}$ and $\left\{T_{k}^{N}=\right.$ $\left.\left(\left|T_{k}^{N}\right|, \tau_{k}^{N}, \varrho_{k}^{N}\right)\right\}$ be controlled $C^{2}$ tube systems for $\left\{M_{k}\right\}$ and $\left\{N_{k}\right\}$ respectively. Let $\varphi: M \rightarrow N$ be a homeomorphism such that $\left.\varphi\right|_{M_{k}}$ is a $C^{2}$ diffeomorphism onto $N_{k}$ for each $k$. We call $\varphi$ compatible with $\left\{T_{k}^{M}\right\}$ and $\left\{T_{k}^{N}\right\}$ if for each $k, \varphi\left(M \cap\left|T_{k}^{M}\right|\right)=N \cap\left|T_{k}^{N}\right|$ and

$$
\tau_{k}^{N} \circ \varphi=\varphi \circ \tau_{k}^{M} \quad \text { and } \quad \varrho_{k}^{N} \circ \varphi=\varrho_{k}^{M} \quad \text { on } M \cap\left|T_{k}^{M}\right| .
$$

Let $f$ and $f_{l}, l=1,2, \ldots$, be semialgebraic $C^{0}$ functions on a semialgebraic set $M$. We say that $f_{l} \rightarrow f$ in the $C^{0}$ topology as $l \rightarrow \infty$ if for any positive semialgebraic $C^{0}$ function $g$ on $M$ there exists $l_{0} \in \mathbb{Z}$ such that $\left|f_{l}-f\right|<g$ for $l \geq l_{0}$. Assume $M$ is a semialgebraic $C^{2}$ manifold and $f_{l}$ and $f$ are semialgebraic $C^{2}$ functions. Then we say that $f_{l} \rightarrow f$ in the $C^{2}$ topology if $f_{l} \rightarrow f$ and the differentials $d f_{l} \rightarrow d f, d d f_{l} \rightarrow d d f$ in the $C^{0}$ topology. In the same way we define the $C^{0}$ topology on the set of semialgebraic $C^{0}$ maps between semialgebraic sets and the $C^{2}$ topology on the set of semialgebraic $C^{2}$ maps between semialgebraic $C^{2}$ manifolds.

The key to the proof of the theorem is the following lemma, which does not hold without the assumption of semialgebraicness nor without the one that $M_{k}$ are semialgebraically diffeomorphic to Euclidean spaces (see $\left[\mathrm{S}_{2}\right.$, p. 238 and Remark II.7.4]).

Lemma (Uniqueness of controlled semialgebraic tube system, $\left[\mathrm{S}_{2}\right.$, Theorem II.7.3]). Let $\left\{M_{k}\right\}$ and $\left\{N_{k}\right\}$ be finite semialgebraic Whitney $C^{2}$ stratifications of locally compact semialgebraic sets $M$ and $N$, respectively, such that all strata are semialgebraically $C^{2}$ diffeomorphic to Euclidean spaces. Let $\left\{T_{k}^{M}\right\}$ and $\left\{T_{k}^{N}\right\}$ be controlled semialgebraic $C^{2}$ tube systems for $\left\{M_{k}\right\}$ and $\left\{N_{k}\right\}$ respectively. Let $\varphi: M \rightarrow N$ be a semialgebraic homeomorphism such that $\left.\varphi\right|_{M_{k}}$ is a $C^{2}$ diffeomorphism onto $N_{k}$ for each $k$. Then shrinking $\left|T_{k}^{M}\right|$ and $\left|T_{k}^{N}\right|$, we can modify $\varphi$ to be compatible with $\left\{T_{k}^{M}\right\}$ and $\left\{T_{k}^{N}\right\}$.

Proof of Theorem. Let $X \subset \mathbb{R}^{n}$. By the usual triangulation theorem for semialgebraic sets we have a finite number of semialgebraic $C^{0}$ imbeddings $\pi_{\alpha}: B^{\alpha} \rightarrow X, \alpha \in A$, such that each $B^{\alpha}$ is the unit ball (always with center 0) in a Euclidean space, $\left.\pi_{\alpha}\right|_{\operatorname{Int} B^{\alpha}}$ is a $C^{\omega}$ imbedding,

$$
\pi_{\alpha}\left(\operatorname{Int} B^{\alpha}\right) \cap \pi_{\alpha^{\prime}}\left(\operatorname{Int} B^{\alpha^{\prime}}\right)=\emptyset \quad \text { for } \alpha \neq \alpha^{\prime} \in A,
$$

$\left\{\pi_{\alpha}\left(\operatorname{Int} B^{\alpha}\right): \alpha \in A\right\}$ is a Whitney stratification of $X$ compatible with $\left\{Y_{i}\right\}$, $\pi_{\alpha}^{-1}\left(\pi_{\alpha^{\prime}}\left(\operatorname{Int} B^{\alpha^{\prime}}\right)\right)$ is a $C^{\omega}$ manifold for $\alpha \in A$ and $\alpha^{\prime} \in A_{\alpha}=\left\{\alpha^{\prime} \in A\right.$ : $\left.\pi_{\alpha^{\prime}}\left(B^{\alpha^{\prime}}\right) \subset \pi_{\alpha}\left(B^{\alpha}\right)\right\}$, the restriction of $\pi_{\alpha}$ to the manifold is a $C^{\omega}$ imbedding, and $\left\{\pi_{\alpha}^{-1}\left(\pi_{\alpha^{\prime}}\left(\operatorname{Int} B^{\alpha^{\prime}}\right)\right): \alpha^{\prime} \in A_{\alpha}\right\}$ is a Whitney stratification of $B^{\alpha}$ for each $\alpha$. We then call $\left\{\pi_{\alpha}: \alpha \in A\right\}$ a semialgebraic $C^{\omega}$ ball decomposition of $X$ (cf. [R-S]). Let the ambient space of $B^{\alpha}$ always be $B^{\alpha}$ itself. 
Set $d_{\alpha}=\operatorname{dim} B^{\alpha}$ and

$$
\begin{gathered}
C_{\alpha}=\pi_{\alpha}\left(B^{\alpha}\right), \quad \partial C_{\alpha}=\pi_{\alpha}\left(\partial B^{\alpha}\right), \quad \operatorname{Int} C_{\alpha}=\pi_{\alpha}\left(\operatorname{Int} B^{\alpha}\right) \quad \text { for } \alpha \in A \\
B_{\alpha^{\prime}}^{\alpha}=\pi_{\alpha}^{-1}\left(C_{\alpha^{\prime}}\right), \quad \partial B_{\alpha^{\prime}}^{\alpha}=\pi_{\alpha}^{-1}\left(\partial C_{\alpha^{\prime}}\right), \\
\operatorname{Int} B_{\alpha^{\prime}}^{\alpha}=\pi_{\alpha}^{-1}\left(\operatorname{Int} C_{\alpha^{\prime}}\right) \text { for } \alpha^{\prime} \in A_{\alpha} .
\end{gathered}
$$

Then $\left\{\operatorname{Int} C_{\alpha}: \alpha \in A\right\}$ and $\left\{\operatorname{Int} B_{\alpha^{\prime}}^{\alpha}: \alpha^{\prime} \in A_{\alpha}\right\}$ for each $\alpha \in A$ are Whitney stratifications of $X$ and $B^{\alpha}$ respectively.

Let $0 * Y$ denote the cone with vertex 0 in some $B^{\alpha}$ and base $Y \subset \partial B^{\alpha}$. Let $\left\{T_{\alpha}=\left(\left|T_{\alpha}\right|, \tau_{\alpha}, \varrho_{\alpha}\right): \alpha \in A\right\}$ and $\left\{T_{\alpha^{\prime}}^{\alpha}=\left(\left|T_{\alpha^{\prime}}^{\alpha}\right|, \tau_{\alpha^{\prime}}^{\alpha}, \varrho_{\alpha^{\prime}}^{\alpha}\right): \alpha^{\prime} \in A_{\alpha}\right\}$ for each $\alpha \in A$ be controlled semialgebraic $C^{2}$ tube systems for $\left\{\operatorname{Int} C_{\alpha}: \alpha \in A\right\}$ and $\left\{\right.$ Int $\left.B_{\alpha^{\prime}}^{\alpha}: \alpha^{\prime} \in A_{\alpha}\right\}$ respectively. Then we can choose the latter so that

$$
\begin{aligned}
& \left(\tau_{\alpha^{\prime}}^{\alpha}\right)^{-1}(x)=\left|T_{\alpha^{\prime}}^{\alpha}\right| \cap 0 *\left(\partial B^{\alpha} \cap\left(\tau_{\alpha^{\prime}}^{\alpha}\right)^{-1}(x)\right) \\
& \qquad \text { for } x \in \operatorname{Int} B_{\alpha^{\prime}}^{\alpha}, \alpha^{\prime} \in A_{\alpha}-\{\alpha\}, \alpha \in A
\end{aligned}
$$

by the usual method of construction of controlled tube systems (see e.g. [G-al]). Moreover, we can modify $\left\{\pi_{\alpha}\right\}$ so that each $\pi_{\alpha}: B^{\alpha} \rightarrow C_{\alpha}$ is compatible with $\left\{T_{\alpha^{\prime}}^{\alpha}: \alpha^{\prime} \in A_{\alpha}\right\}$ and $\left\{T_{\alpha^{\prime}}: \alpha^{\prime} \in A_{\alpha}\right\}$ by the lemma. Here $\left.\pi_{\alpha}\right|_{\text {Int } B_{\alpha^{\prime}}^{\alpha}}$ are no longer of class $C^{\omega}$ but $C^{2}$. Hence $\left\{\pi_{\alpha}: \alpha \in A\right\}$ is called a semialgebraic $C^{2}$ ball decomposition of $X$. By loosening the condition of compatibility we will $C^{\omega}$ smooth later.

In the following arguments we subdivide $\left\{\operatorname{Int} C_{\alpha}\right\}$, which works by the following fact:

(*) Let $\pi_{\alpha}, A, A_{\alpha}, B^{\alpha}, B_{\alpha^{\prime}}^{\alpha}, T_{\alpha}$ and $T_{\alpha^{\prime}}^{\alpha}$ be given as above (i.e., $\left\{\pi_{\alpha}\right\}$ is a semialgebraic $C^{2}$ ball decomposition of $X,(1)$ is satisfied and $\pi_{\alpha}$ are compatible). Let $\left\{\pi_{\widetilde{\alpha}}: B^{\widetilde{\alpha}} \rightarrow X: \widetilde{\alpha} \in \widetilde{A}\right\}$ be a second semialgebraic $C^{2}$ ball decomposition of $X$ but without tube systems as yet. Define $\widetilde{A}_{\widetilde{\alpha}}, B_{\widetilde{\alpha}^{\prime}}^{\widetilde{\alpha}}$ and $C_{\widetilde{\alpha}}$ in the same way. Let $m \in \mathbb{Z}$. Assume

$$
\begin{aligned}
A \cap \widetilde{A} & =\left\{\alpha \in A: d_{\alpha}>m\right\}=\left\{\widetilde{\alpha} \in \widetilde{A}: d_{\widetilde{\alpha}}>m\right\}, \\
B^{\alpha} & =B^{\widetilde{\alpha}} \quad \text { for } \alpha=\widetilde{\alpha} \in A \cap \widetilde{A}
\end{aligned}
$$

the maps $\pi_{\alpha}: B^{\alpha} \rightarrow C_{\alpha}$ and $\pi_{\widetilde{\alpha}}: B^{\widetilde{\alpha}} \rightarrow C_{\widetilde{\alpha}}$ coincide for $\alpha=\widetilde{\alpha} \in A \cap \widetilde{A}$, and $\left\{\operatorname{Int} C_{\widetilde{\alpha}}: \widetilde{\alpha} \in \widetilde{A}, d_{\widetilde{\alpha}} \leq m\right\}$ is compatible with $\left\{\operatorname{Int} C_{\alpha}: \alpha \in A, d_{\alpha} \leq m\right\}$. Then fixing $\pi_{\widetilde{\alpha}}$ for $\widetilde{\alpha} \in \widetilde{A}$ with $d_{\widetilde{\alpha}}>m$ and $\operatorname{Im} \pi_{\widetilde{\alpha}}, d_{\widetilde{\alpha}} \leq m$, and modifying only $\pi_{\widetilde{\alpha}}, d_{\widetilde{\alpha}} \leq m$, we have controlled semialgebraic $C^{2}$ tube systems $\left\{T_{\widetilde{\alpha}}\right.$ : $\widetilde{\alpha} \in \widetilde{A}\}$ for $\left\{\operatorname{Int} C_{\widetilde{\alpha}}: \widetilde{\alpha} \in \widetilde{A}\right\}$ and $\left\{T_{\widetilde{\alpha}^{\prime}}^{\widetilde{\alpha}}: \widetilde{\alpha}^{\prime} \in \widetilde{A}_{\widetilde{\alpha}}\right\}$ for $\left\{\operatorname{Int} B_{\widetilde{\alpha}^{\prime}}^{\widetilde{\alpha}}: \widetilde{\alpha}^{\prime} \in \widetilde{A}_{\widetilde{\alpha}}\right\}$ for each $\widetilde{\alpha} \in \widetilde{A}$ with the same properties as $\left\{T_{\alpha}\right\}$ and $\left\{T_{\widetilde{\alpha}^{\prime}}^{\widetilde{\alpha}}\right\}$-by (1) and compatibility of $\pi_{\widetilde{\alpha}}$ - such that

$$
T_{\widetilde{\alpha}}= \begin{cases}T_{\alpha} & \text { for } \widetilde{\alpha}=\alpha \in A \cap \widetilde{A}, \\ \left.T_{\alpha}\right|_{\left|T_{\widetilde{\alpha}}\right|} & \text { for } \alpha \in A, \widetilde{\alpha} \in \widetilde{A} \text { with } C_{\widetilde{\alpha}} \subset C_{\alpha}, d_{\alpha}=d_{\widetilde{\alpha}}=m,\end{cases}
$$


and for each $\widetilde{\alpha}=\alpha \in A \cap \widetilde{A}$,

$$
T_{\widetilde{\alpha}^{\prime}}^{\widetilde{\alpha}}= \begin{cases}T_{\alpha^{\prime}}^{\alpha} & \text { for } \widetilde{\alpha}=\alpha \in A \cap \widetilde{A}, \widetilde{\alpha}^{\prime}=\alpha^{\prime} \in A_{\alpha} \cap \widetilde{A}_{\widetilde{\alpha}} \\ T_{\alpha^{\prime}\left|T_{\widetilde{\alpha}^{\prime}}^{\alpha}\right|}^{\alpha} & \text { for } \widetilde{\alpha}=\alpha \in A \cap \widetilde{A}, \alpha^{\prime} \in A_{\alpha}, \widetilde{\alpha}^{\prime} \in \widetilde{A}_{\widetilde{\alpha}} \\ & \text { with } B_{\widetilde{\alpha}^{\prime}}^{\widetilde{\alpha}} \subset B_{\alpha^{\prime}}^{\alpha}, d_{\alpha^{\prime}}=d_{\widetilde{\alpha}^{\prime}}=m .\end{cases}
$$

Proof of $(*)$. It suffices to find a controlled semialgebraic $C^{2}$ tube system $\left\{T_{\widetilde{\alpha}}: \widetilde{\alpha} \in \widetilde{A}\right\}$ for $\left\{\operatorname{Int} C_{\widetilde{\alpha}}: \widetilde{\alpha} \in \widetilde{A}\right\}$ such that (2) holds and

$$
\begin{aligned}
& \tau_{\widetilde{\alpha}}=\tau_{\widetilde{\alpha}} \circ \tau_{\alpha}, \quad \varrho_{\widetilde{\alpha}}=\varrho_{\alpha}+\varrho_{\widetilde{\alpha}} \circ \tau_{\alpha} \text { on }\left|T_{\widetilde{\alpha}}\right| \\
& \quad \text { for } \alpha \in A, \widetilde{\alpha} \in \widetilde{A} \text { with } \operatorname{Int} C_{\widetilde{\alpha}} \subset \operatorname{Int} C_{\alpha}, d_{\widetilde{\alpha}}<m .
\end{aligned}
$$

Indeed, assume there exists such $\left\{T_{\widetilde{\alpha}}\right\}$. Using it, we need to construct $\left\{T_{\widetilde{\alpha}^{\prime}}^{\widetilde{\alpha}}: \widetilde{\alpha}^{\prime} \in \widetilde{A}_{\widetilde{\alpha}}\right\}$ for each $\widetilde{\alpha} \in \widetilde{A}$ as required in $(*)$.

For $\widetilde{\alpha} \in A \cap \widetilde{A}$ and $\widetilde{\alpha}^{\prime} \in \widetilde{A}_{\widetilde{\alpha}}$, define $T_{\widetilde{\alpha}^{\prime}}^{\widetilde{\alpha}}$ by

$$
\left|T_{\widetilde{\alpha}^{\prime}}^{\widetilde{\alpha}}\right|=\pi_{\widetilde{\alpha}}^{-1}\left(\left|T_{\widetilde{\alpha}^{\prime}}\right|\right), \quad \tau_{\widetilde{\alpha}^{\prime}}^{\widetilde{\alpha}}=\pi_{\widetilde{\alpha}}^{-1} \circ \tau_{\widetilde{\alpha}^{\prime}} \circ \pi_{\widetilde{\alpha}}, \quad \varrho_{\widetilde{\alpha}^{\prime}}^{\widetilde{\alpha}}=\varrho_{\widetilde{\alpha}^{\prime}} \circ \pi_{\widetilde{\alpha}} .
$$

Then for each $\widetilde{\alpha} \in A \cap \widetilde{A},\left\{T_{\widetilde{\alpha}^{\prime}}^{\widetilde{\alpha}}: \widetilde{\alpha}^{\prime} \in \widetilde{A}_{\widetilde{\alpha}}\right\}$ is a controlled semialgebraic $C^{2}$ tube system for $\left\{\right.$ Int $\left.B_{\widetilde{\alpha}^{\prime}}^{\widetilde{\alpha}}: \widetilde{\alpha}^{\prime} \in \widetilde{A}_{\widetilde{\alpha}}\right\}$. Indeed, $\left|T_{\widetilde{\alpha}^{\prime}}^{\widetilde{\alpha}}\right|$ is a semialgebraic neighborhood of Int $B_{\widetilde{\alpha}^{\prime}}^{\widetilde{\alpha}}$ in $B^{\widetilde{\alpha}} ; \tau_{\widetilde{\alpha}^{\prime}}^{\widetilde{\alpha}}:\left|T_{\widetilde{\alpha}^{\prime}}^{\widetilde{\alpha}}\right| \rightarrow \operatorname{Int} B_{\widetilde{\alpha}^{\prime}}^{\widetilde{\alpha}}$ is a semialgebraic retraction and of class $C^{2}$ because $\left.\pi_{\widetilde{\alpha}}^{-1}\right|_{\operatorname{Int} C_{\widetilde{\alpha}^{\prime}}}$ is of class $C^{2}$ and because

$$
\tau_{\widetilde{\alpha}^{\prime}} \circ \pi_{\widetilde{\alpha}} \stackrel{(3)}{=} \tau_{\widetilde{\alpha}^{\prime}} \circ \tau_{\alpha^{\prime}} \circ \pi_{\alpha} \stackrel{\text { compatibility of } \pi_{\alpha}}{=} \tau_{\widetilde{\alpha}^{\prime}} \circ \pi_{\alpha} \circ \tau_{\alpha^{\prime}}^{\alpha}
$$

where $\alpha \in A$ and $\alpha^{\prime} \in A_{\alpha}$ with $\alpha=\widetilde{\alpha}$ Int $C_{\widetilde{\alpha}^{\prime}} \subset \operatorname{Int} C_{\alpha^{\prime}}$, and because $\left.\pi_{\alpha}\right|_{\operatorname{Int} B_{\alpha^{\prime}}^{\alpha}}: \operatorname{Int} B_{\alpha^{\prime}}^{\alpha} \rightarrow \operatorname{Int} C_{\alpha^{\prime}}$ is a $C^{2}$ diffeomorphism; $\varrho_{\widetilde{\alpha}^{\prime}}^{\widetilde{\alpha}}$ is a nonnegative semialgebraic $C^{0}$ function on $\left|T_{\widetilde{\alpha}^{\prime}}^{\widetilde{\alpha}}\right| ;\left(\varrho_{\widetilde{\alpha}^{\prime}}^{\widetilde{\alpha}}\right)^{-1}(0)=\operatorname{Int} B_{\widetilde{\alpha}^{\prime}}^{\widetilde{\alpha}} ; \varrho_{\widetilde{\alpha}^{\prime}}^{\widetilde{\alpha}}$ is of class $C^{2}$ and each $x \in \operatorname{Int} B_{\widetilde{\alpha}^{\prime}}^{\widetilde{\alpha}}$ is a unique and nondegenerate critical point of $\left.\varrho_{\widetilde{\alpha}^{\prime}}^{\widetilde{\alpha}}\right|_{\left(\tau_{\widetilde{\alpha}^{\prime}}^{\widetilde{\alpha}}\right)^{-1}(x)}$ because

$$
\begin{aligned}
\varrho_{\widetilde{\alpha}^{\prime}}^{\widetilde{\alpha}} & =\varrho_{\widetilde{\alpha}^{\prime}} \circ \pi_{\widetilde{\alpha}} \stackrel{(3)}{=} \varrho_{\alpha^{\prime}} \circ \pi_{\widetilde{\alpha}}+\varrho_{\widetilde{\alpha}^{\prime}} \circ \tau_{\alpha^{\prime}} \circ \pi_{\widetilde{\alpha}} \\
& =\varrho_{\alpha^{\prime}} \circ \pi_{\alpha}+\left(\varrho_{\widetilde{\alpha}^{\prime}} \mid \operatorname{Int} C_{\alpha^{\prime}}\right) \circ \tau_{\alpha^{\prime}} \circ \pi_{\alpha} \\
\text { compatibility of } \pi_{\alpha} & \stackrel{=}{=} \varrho_{\alpha^{\prime}}^{\alpha}+\left(\varrho_{\widetilde{\alpha}^{\prime}} \mid \operatorname{Int} C_{\alpha^{\prime}}\right) \circ \pi_{\alpha} \circ \tau_{\alpha^{\prime}}^{\alpha} .
\end{aligned}
$$

Moreover, by (4), $\pi_{\widetilde{\alpha}}$ is compatible with $\left\{T_{\widetilde{\alpha}^{\prime}}^{\widetilde{\alpha}}: \widetilde{\alpha}^{\prime} \in \widetilde{A}_{\widetilde{\alpha}}\right\}$ and $\left\{T_{\widetilde{\alpha}^{\prime}}: \widetilde{\alpha}^{\prime} \in \widetilde{A}_{\widetilde{\alpha}}\right\}$ for each $\widetilde{\alpha} \in A \cap \widetilde{A}$, and clearly (2)' is satisfied by (2) since $\pi_{\alpha}$ is compatible with $\left\{T_{\alpha^{\prime}}^{\alpha}: \alpha^{\prime} \in A_{\alpha}\right\}$ and $\left\{T_{\alpha^{\prime}}: \alpha^{\prime} \in A_{\alpha}\right\}$.

For $\widetilde{\alpha} \in \widetilde{A}-A$, i.e., $d_{\widetilde{\alpha}} \leq m$, let $\left\{T_{\widetilde{\alpha}^{\prime}}^{\widetilde{\alpha}}: \widetilde{\alpha}^{\prime} \in \widetilde{A}_{\widetilde{\alpha}}\right\}$ be an arbitrary controlled semialgebraic $C^{2}$ tube system for $\left\{\operatorname{Int} B_{\widetilde{\alpha}^{\prime}}^{\widetilde{\alpha}}: \widetilde{\alpha}^{\prime} \in \widetilde{A}_{\widetilde{\alpha}}\right\}$ with (1). Then by the Lemma we can modify $\pi_{\widetilde{\alpha}}$ and assume it is compatible with $\left\{T_{\widetilde{\alpha}^{\prime}}^{\widetilde{\alpha}}: \widetilde{\alpha}^{\prime} \in \widetilde{A}_{\widetilde{\alpha}}\right\}$ and $\left\{T_{\widetilde{\alpha}^{\prime}}: \widetilde{\alpha}^{\prime} \in \widetilde{A}_{\widetilde{\alpha}}\right\}$. 
Thus $\left\{T_{\widetilde{\alpha}^{\prime}}^{\widetilde{\alpha}}\right\}$ together with $\left\{T_{\widetilde{\alpha}}\right\}$ fulfills the requirements in $(*)$, and hence we will construct only $\left\{T_{\widetilde{\alpha}}\right\}$ with (2) and (3).

Order the elements of $\widetilde{A}$ as $\widetilde{\alpha}_{1}, \widetilde{\alpha}_{2}, \ldots$ so that $d_{\widetilde{\alpha}_{k}} \leq d_{\widetilde{\alpha}_{k+1}}$ for any $k$. Let $m^{\prime} \in \mathbb{Z}$. Assume by induction we have a controlled semialgebraic $C^{2}$ tube system $\left\{T_{\widetilde{\alpha}_{1}}, \ldots, T_{\widetilde{\alpha}_{m^{\prime}-1}}\right\}$ for $\left\{\operatorname{Int} C_{\widetilde{\alpha}_{1}}, \ldots, \operatorname{Int} C_{\widetilde{\alpha}_{m^{\prime}-1}}\right\}$ with (2) and (3). Let $\alpha_{k} \in A$ be such that Int $C_{\widetilde{\alpha}_{k}} \subset \operatorname{Int} C_{\alpha_{k}}$ for each $k$. Then we only need to find a semialgebraic $C^{2}$ tube $T_{\widetilde{\alpha}_{m^{\prime}}}$ about Int $C_{\widetilde{\alpha}_{m^{\prime}}}$ such that (2) and (3) hold for $\alpha=\alpha_{m^{\prime}}$ and $\widetilde{\alpha}=\widetilde{\alpha}_{m^{\prime}}$, and

$$
\begin{aligned}
& \tau_{\widetilde{\alpha}_{k}} \circ \tau_{\widetilde{\alpha}_{m^{\prime}}} \circ \tau_{\alpha_{m^{\prime}}}=\tau_{\widetilde{\alpha}_{k}} \circ \tau_{\alpha_{m^{\prime}}}, \\
& \varrho_{\widetilde{\alpha}_{k}} \circ \tau_{\widetilde{\alpha}_{m^{\prime}}} \circ \tau_{\alpha_{m^{\prime}}}=\varrho_{\widetilde{\alpha}_{k}} \circ \tau_{\alpha_{m^{\prime}}} \quad \text { for } k<m^{\prime}
\end{aligned}
$$

because $\left\{T_{\widetilde{\alpha}_{m^{\prime}}}, T_{\widetilde{\alpha}_{k}}\right\}$ is controlled for each $k<m^{\prime}$ as follows:

$$
\begin{array}{r}
\tau_{\widetilde{\alpha}_{k}} \circ \tau_{\widetilde{\alpha}_{m^{\prime}}} \stackrel{(3)}{=} \tau_{\widetilde{\alpha}_{k}} \circ \tau_{\widetilde{\alpha}_{m^{\prime}}} \circ \tau_{\alpha_{m^{\prime}}} \stackrel{(5)}{=} \tau_{\widetilde{\alpha}_{k}} \circ \tau_{\alpha_{m^{\prime}}} \stackrel{(3)}{=} \tau_{\widetilde{\alpha}_{k}} \circ \tau_{\alpha_{k}} \circ \tau_{\alpha_{m^{\prime}}} \\
\text { controlledness of }\left\{T_{\alpha}\right\} \\
\varrho_{\widetilde{\alpha}_{k}} \circ \tau_{\widetilde{\alpha}_{m^{\prime}}} \stackrel{(3)}{=} \varrho_{\widetilde{\alpha}_{k}} \circ \tau_{\alpha_{\alpha_{k}}} \stackrel{(3)}{=} \tau_{\widetilde{\alpha}_{m^{\prime}}} \circ \tau_{\alpha_{m^{\prime}}}, \\
\stackrel{(5)}{=} \varrho_{\widetilde{\alpha}_{k}} \circ \tau_{\alpha_{m^{\prime}}} \stackrel{(3)}{=} \varrho_{\alpha_{k}} \circ \tau_{\alpha_{m^{\prime}}}+\varrho_{\widetilde{\alpha}_{k}} \circ \tau_{\alpha_{k}} \circ \tau_{\alpha_{m^{\prime}}} \\
\stackrel{\left(\text { controlledness of }\left\{T_{\alpha}\right\}\right.}{=} \varrho_{\alpha_{k}}+\varrho_{\widetilde{\alpha}_{k}} \circ \tau_{\alpha_{k}} \stackrel{(3)}{=} \varrho_{\widetilde{\alpha}_{k}} .
\end{array}
$$

The construction of $T_{\widetilde{\alpha}_{m^{\prime}}}$ is easy. If $d_{\widetilde{\alpha}_{m^{\prime}}} \geq m, T_{\widetilde{\alpha}_{m^{\prime}}}$ is defined by (2). Then (5) is clearly satisfied. Assume $d_{\widetilde{\alpha}_{m^{\prime}}}<m$. Let $\left\{T_{\widetilde{\alpha}_{m^{\prime}}}^{\prime m^{\prime}}=\left(\mid T_{\widetilde{\alpha}_{m^{\prime}}}^{\prime}, \tau_{\widetilde{\alpha}_{m^{\prime}}}^{\prime}, \varrho_{\widetilde{\alpha}_{m^{\prime}}}^{\prime}\right)\right.$, $\left.T_{\widetilde{\alpha}_{k}}: k<m^{\prime}\right\}$ be a controlled semialgebraic $C^{2}$ tube system for $\left\{C_{\widetilde{\alpha}_{k}}\right.$ : $\left.k \leq m^{\prime}\right\}$, whose existence is shown in the usual construction of a controlled tube system (see, e.g., [G-al]). (Here we shrink $\left|T_{\widetilde{\alpha}_{k}}\right|, k<m^{\prime}$, if necessary.) Set

$\left|T_{\widetilde{\alpha}_{m^{\prime}}}\right|=\left|T_{\alpha_{m^{\prime}}}\right| \cap\left|T_{\widetilde{\alpha}_{m^{\prime}}}^{\prime}\right|, \quad \tau_{\widetilde{\alpha}_{m^{\prime}}}=\tau_{\widetilde{\alpha}_{m^{\prime}}}^{\prime} \circ \tau_{\alpha_{m^{\prime}}}, \quad \varrho_{\widetilde{\alpha}_{m^{\prime}}}=\varrho_{\alpha_{m^{\prime}}}+\varrho_{\widetilde{\alpha}_{m^{\prime}}}^{\prime} \circ \tau_{\alpha_{m^{\prime}}}$.

Then (3) and (5) are satisfied. Thus (*) holds.

Let $\left\{\pi_{\alpha}: \alpha \in A\right\}$ be again a semialgebraic $C^{2}$ ball decomposition of $X$ with controlled semialgebraic $C^{2}$ tube systems $\left\{T_{\alpha}: \alpha \in A\right\}$ and $\left\{T_{\alpha^{\prime}}^{\alpha}\right.$ : $\left.\alpha^{\prime} \in A_{\alpha}\right\}$ for each $\alpha \in A$ such that (1) holds and $\pi_{\alpha}$ is compatible with $\left\{T_{\alpha^{\prime}}^{\alpha}: \alpha^{\prime} \in A_{\alpha}\right\}$ and $\left\{T_{\alpha^{\prime}}: \alpha^{\prime} \in A_{\alpha}\right\}$. We show good properties of such $\left\{\pi_{\alpha}\right\},\left\{T_{\alpha}\right\}$ and $\left\{T_{\alpha^{\prime}}^{\alpha}\right\}$. Set

$$
\begin{gathered}
0 *_{\alpha} Y=\pi_{\alpha}\left(0 * \pi_{\alpha}^{-1}(Y)\right) \quad \text { for } Y \subset \partial C_{\alpha}, \\
\mathcal{J}=\left\{\left(\alpha_{1}, \ldots, \alpha_{l}\right) \in A^{l}: l \in \mathbb{N}, \alpha_{1} \in A_{\alpha_{2}}, \ldots, \alpha_{l-1} \in A_{\alpha_{l}}, \alpha_{1} \neq \cdots \neq \alpha_{l}\right\}, \\
U_{J}=\operatorname{Int} 0 *_{\alpha_{l}}\left(\cdots *_{\alpha_{3}}\left(0 *_{\alpha_{2}} C_{\alpha_{1}}\right) \cdots\right) \quad \text { for } J=\left(\alpha_{1}, \ldots, \alpha_{l}\right) \in \mathcal{J}, l>1, \\
U_{x, J}=\operatorname{Int} 0 *_{\alpha_{l}}\left(\cdots *_{\alpha_{3}}\left(0 *_{\alpha_{2}} x\right) \cdots\right) \quad \text { for same } J \text { and } x \in \operatorname{Int} C_{\alpha_{1}}, \\
U_{\alpha}=\operatorname{Int} C_{\alpha}, \quad U_{x, \alpha}=x \quad \text { for } \alpha \in A \text { and } x \in \operatorname{Int} C_{\alpha},
\end{gathered}
$$

where $0 *_{\alpha_{l}}\left(\cdots *_{\alpha_{3}}\left(0 *_{\alpha_{2}} C_{\alpha_{1}}\right) \cdots\right)$ and $0 *_{\alpha_{l}}\left(\cdots *_{\alpha_{3}}\left(0 *_{\alpha_{2}} x\right) \cdots\right)$ are semialgebraically homeomorphic to balls of dimension $d_{\alpha_{1}}+l-1$ and $l-1$, respec- 
tively, and Int stands for open balls. Note that $U_{J}$ and $U_{x, J}$ are $C^{2}$ manifolds. For $J=\left(\alpha_{1}, \ldots, \alpha_{l}\right) \in \mathcal{J}$, let $p_{J}: U_{J} \rightarrow \operatorname{Int} C_{\alpha_{1}}$ denote the semialgebraic $C^{2}$ submersion such that $x \in U_{p_{J}(x), J}$. We also define $U_{J}^{\alpha} \subset B^{\alpha}, U_{x, J}^{\alpha} \subset B^{\alpha}$ and $p_{J}^{\alpha}: U_{J}^{\alpha} \rightarrow \operatorname{Int} B_{\alpha_{1}}^{\alpha}$ to be $\pi_{\alpha}^{-1}\left(U_{J}\right), \pi_{\alpha}^{-1}\left(U_{\pi_{\alpha}(x), J}\right)$ and $\pi_{\alpha}^{-1} \circ p_{J} \circ \pi_{\alpha}$, respectively, for $J=\left(\alpha_{1}, \ldots, \alpha_{l}\right) \in \mathcal{J}, \alpha \in A$ and $x \in \operatorname{Int} B_{\alpha_{1}}^{\alpha}$ with $\alpha_{l} \in A_{\alpha}$. Note that $U_{J}, p_{J}, U_{J}^{\alpha}$ and $p_{J}^{\alpha}$ depend only on $\left\{\pi_{\alpha}\right\}$ but not on $\left\{T_{\alpha}\right\},\left\{T_{\alpha^{\prime}}^{\alpha}\right\}$.

Then by (1) and compatibility of $\left\{\pi_{\alpha}: \alpha \in A\right\}$ we have

$$
p_{J}=\tau_{\alpha_{1}} \quad \text { on } U_{J} \cap\left|T_{\alpha_{1}}\right|, \quad p_{J}^{\alpha}=\tau_{\alpha_{1}}^{\alpha} \quad \text { on } U_{J}^{\alpha} \cap\left|T_{\alpha_{1}}^{\alpha}\right| .
$$

Moreover, as shown below, we can suppose the following condition (7) is satisfied, which will allow a "derived" subdivision of $\left\{\operatorname{Int} C_{\alpha}\right\}$ also to be a Whitney stratification.

(7) $\left\{U_{J}\right.$, Int $\left.C_{\alpha_{1}}\right\}$ (resp. $\left\{U_{J}^{\alpha}\right.$, Int $\left.B_{\alpha_{1}}^{\alpha}\right\}$ ) satisfies the Whitney condition.

Note that (7) depends on $C_{\alpha_{1}}$ but not on $\pi_{\alpha_{1}}$, hence fixing $C_{\alpha_{1}}$ we can change $\pi_{\alpha_{1}}$ arbitrarily when (7) is satisfied and when we need to keep the property, and that (6) and (7) imply that:

$\left\{p_{J}^{-1}\left(M_{1}\right), M_{2}\right\}$ (resp. $\left.\left(\left\{p_{J}^{\alpha}\right)^{-1}\left(M_{1}\right), M_{2}\right\}\right)$ is a Whitney stratification for a Whitney $C^{2}$ stratification $\left\{M_{1}, M_{2}\right\}$ in $\operatorname{Int} C_{\alpha_{1}}\left(\operatorname{resp}\right.$. Int $\left.B_{\alpha_{1}}^{\alpha}\right)$ or for a $C^{2}$ submanifold $M_{1}=M_{2}$ of Int $C_{\alpha_{1}}\left(\right.$ resp. Int $\left.B_{\alpha_{1}}^{\alpha}\right)$.

We modify $\left\{\pi_{\alpha}\right\}$ so that (7) is satisfied by downward induction. Let $0 \leq m \leq n$ be an integer. Assume we have a semialgebraic $C^{2}$ ball decomposition $\left\{\pi_{\alpha}: \alpha \in A\right\}$ of $X$ and controlled semialgebraic $C^{2}$ tube systems $\left\{T_{\alpha}: \alpha \in A\right\}$ for $\left\{C_{\alpha}: \alpha \in A\right\}$ and $\left\{T_{\alpha^{\prime}}^{\alpha}: \alpha^{\prime} \in A_{\alpha}\right\}$ for $\left\{B_{\alpha^{\prime}}^{\alpha}: \alpha^{\prime} \in A_{\alpha}\right\}$ for each $\alpha \in A$ such that (1) is satisfied for any $\alpha \in A$ and $\alpha^{\prime} \in A_{\alpha}$, any $\pi_{\alpha}$ is compatible with $\left\{T_{\alpha^{\prime}}^{\alpha}: \alpha^{\prime} \in A_{\alpha}\right\}$ and $\left\{T_{\alpha^{\prime}}: \alpha^{\prime} \in A_{\alpha}\right\}$, and (7) is satisfied for any $J=\left(\alpha_{1}, \ldots, \alpha_{l}\right) \in \mathcal{J}$ with $l>1$ and $d_{\alpha_{1}}>m$. Then we need to find a semialgebraic $C^{2}$ ball decomposition $\left\{\pi_{\widetilde{\alpha}}: \widetilde{\alpha} \in \widetilde{A}\right\}$ and controlled semialgebraic $C^{2}$ tube systems $\left\{T_{\widetilde{\alpha}}: \widetilde{\alpha} \in \widetilde{A}\right\}$ for $\left\{C_{\widetilde{\alpha}}: \widetilde{\alpha} \in \widetilde{A}\right\}$ and $\left\{T_{\widetilde{\alpha}^{\prime}}^{\widetilde{\alpha}}: \widetilde{\alpha}^{\prime} \in \widetilde{A}_{\widetilde{\alpha}}\right\}$ for $\left\{B_{\widetilde{\alpha}^{\prime}}^{\widetilde{\alpha}}: \widetilde{\alpha}^{\prime} \in \widetilde{A}_{\widetilde{\alpha}}\right\}$ with the same properties as $\left\{\pi_{\alpha}\right\},\left\{T_{\alpha}\right\}$ and $\left\{T_{\alpha^{\prime}}^{\alpha}\right\}$ and, moreover, such that $(7)$ is satisfied also for $\widetilde{J}=\left(\widetilde{\alpha}_{1}, \ldots, \widetilde{\alpha}_{l}\right) \in \widetilde{\mathcal{J}}$ with $l>1$ and $d_{\widetilde{\alpha}_{1}}=m$, where $C_{\widetilde{\alpha}}, \widetilde{A}_{\widetilde{\alpha}}, B_{\widetilde{\alpha}^{\prime}}^{\widetilde{\alpha}}$ and $\widetilde{\mathcal{J}}$ are defined as before. We further require, as in $(*)$,

$$
\begin{aligned}
A \cap \widetilde{A} & =\left\{\alpha \in A: d_{\alpha}>m\right\}=\left\{\widetilde{\alpha} \in \widetilde{A}: d_{\widetilde{\alpha}}>m\right\}, \\
B^{\alpha} & =B^{\widetilde{\alpha}} \quad \text { for } \alpha=\widetilde{\alpha} \in A \cap \widetilde{A},
\end{aligned}
$$

$\pi_{\alpha}: B^{\alpha} \rightarrow C_{\alpha}$ and $\pi_{\widetilde{\alpha}}: B^{\widetilde{\alpha}} \rightarrow C_{\widetilde{\alpha}}$ coincide for $\alpha=\widetilde{\alpha} \in A \cap \widetilde{A}$, and $\left\{\operatorname{Int} C_{\widetilde{\alpha}}\right.$ :

$\left.\widetilde{\alpha} \in \widetilde{A}, d_{\widetilde{\alpha}} \leq m\right\}$ is compatible with $\left\{\operatorname{Int} C_{\alpha}: \alpha \in A, d_{\alpha} \leq m\right\}$. The construction of such $\left\{\pi_{\widetilde{\alpha}}\right\},\left\{T_{\widetilde{\alpha}}\right\}$ and $\left\{T_{\widetilde{\alpha}^{\prime}}^{\widetilde{\alpha}}\right\}$ is clear by $(*)$ and the following well known fact: 
FACT. Fix $J=\left(\alpha_{1}, \ldots, \alpha_{l}\right) \in \mathcal{J}$ and $\alpha \in A$ with $l>1, \alpha_{l} \in A_{\alpha}$ and $d_{\alpha_{1}}=m$. The subset of $\operatorname{Int} C_{\alpha_{1}}$ (resp. Int $\left.B_{\alpha_{1}}^{\alpha}\right)$ consisting of points where $\left\{U_{J}\right.$, Int $\left.C_{\alpha_{1}}\right\}$ (resp. $\left\{U_{J}^{\alpha}\right.$, Int $\left.\left.B_{\alpha_{1}}^{\alpha}\right\}\right)$ does not satisfy the Whitney condition is semialgebraic and of dimension smaller than $m$.

Next we will $C^{\omega}$ smooth $\left.\pi_{\alpha}\right|_{\operatorname{Int} B^{\alpha}}$. We assume $X$ is the unit ball in $\mathbb{R}^{n}$ for simplicity of notation and without loss of generality. Preparatory to smoothing, set

$$
\varepsilon *_{\alpha} Y=\pi_{\alpha}\left\{t \pi_{\alpha}^{-1}(x): 1-\varepsilon \leq t<1, x \in Y\right\}
$$

for $\varepsilon \in] 0,1\left[, \alpha \in A, Y \subset \partial C_{\alpha}\right.$,

$$
\begin{aligned}
U_{J}(\varepsilon)=\left\{\varepsilon_{l} *_{\alpha_{l}}\left(\cdots *_{\alpha_{3}}\left(\varepsilon_{2} *_{\alpha_{2}} \operatorname{Int} C_{\alpha_{1}}\right) \cdots\right):\right. \\
\left.\varepsilon_{2}, \ldots, \varepsilon_{l} \in\right] 0,1\left[, \varepsilon_{2}+\cdots+\varepsilon_{l}=\varepsilon\right\}
\end{aligned}
$$

for $J=\left(\alpha_{1}, \ldots, \alpha_{l}\right) \in \mathcal{J}, l>1$,

$$
\begin{aligned}
U_{\alpha}(\varepsilon) & =\operatorname{Int} C_{\alpha}, \\
W_{\alpha_{1}}(\varepsilon) & =\bigcup_{J=\left(\alpha_{1}, \ldots, \alpha_{l}\right) \in \mathcal{J}} U_{J}(\varepsilon) \quad \text { for each } \alpha_{1} \in A,
\end{aligned}
$$

and define naturally $U_{J}^{\alpha}(\varepsilon)$ and $W_{\alpha_{1}}^{\alpha}(\varepsilon)$ in $B^{\alpha}$. Then for each $\alpha \in A$ and $J=\left(\alpha_{1}, \ldots, \alpha_{l}\right) \in \mathcal{J}$ with $\alpha_{l} \in A_{\alpha}$, we have $U_{J}(\varepsilon) \subset U_{J}\left(\varepsilon^{\prime}\right)\left(\operatorname{resp} . U_{J}^{\alpha}(\varepsilon) \subset\right.$ $\left.U_{J}^{\alpha}\left(\varepsilon^{\prime}\right)\right)$ for $0<\varepsilon<\varepsilon^{\prime}<1, \bigcup_{\varepsilon \in] 0,1[} U_{J}(\varepsilon)=U_{J}\left(\operatorname{resp} . \bigcup_{\varepsilon \in] 0,1[} U_{J}^{\alpha}(\varepsilon)=U_{J}^{\alpha}\right)$, $W_{\alpha_{1}}(\varepsilon)$ (resp. $\left.W_{\alpha_{1}}^{\alpha}(\varepsilon)\right)$ is a neighborhood of $\operatorname{Int} C_{\alpha_{1}}$ in $X$ (resp. $\operatorname{Int} B_{\alpha_{1}}^{\alpha}$ in $B^{\alpha}$ ) called the $\varepsilon$-neighborhood of $\operatorname{Int} C_{\alpha_{1}}$ (resp. Int $B_{\alpha_{1}}^{\alpha}$ ), and the map $q_{\alpha_{1}}: W_{\alpha_{1}}(\varepsilon) \rightarrow \operatorname{Int} C_{\alpha_{1}}$ (resp. $q_{\alpha_{1}}^{\alpha}: W_{\alpha_{1}}^{\alpha}(\varepsilon) \rightarrow \operatorname{Int} B_{\alpha_{1}}^{\alpha}$ ) defined to be $p_{J}$ on $U_{J}(\varepsilon)$ (resp. $p_{J}^{\alpha}$ on $U_{J}^{\alpha}(\varepsilon)$ ) is proper. (If we define $W_{\alpha_{1}}(\varepsilon)$ and $q_{\alpha_{1}}$ with $U_{J}$ in place of $U_{J}(\varepsilon)$, then $q_{\alpha_{1}}$ is not proper for $l>1$. This is the reason why we apply $U_{J}(\varepsilon)$ and not $U_{J}$.)

For smoothing we drop compatibility of $\pi_{\alpha}$ and weaken (6) as follows:

$(6)^{\prime} \quad$ For $\alpha \in A$ and $\alpha_{1} \in A_{\alpha}, q_{\alpha_{1}}: W_{\alpha_{1}}(\varepsilon) \rightarrow \operatorname{Int} C_{\alpha_{1}}$ (resp. $q_{\alpha_{1}}^{\alpha}: W_{\alpha_{1}}^{\alpha}(\varepsilon) \rightarrow$ Int $\left.B_{\alpha_{1}}^{\alpha}\right)$ is a submersive $C^{2}$ retraction for some $\varepsilon$.

Namely, we are in the situation that $\left\{\pi_{\alpha}: \alpha \in A\right\}$ is a semialgebraic $C^{2}$ ball decomposition of $X,\left\{T_{\alpha}: \alpha \in A\right\}$ and $\left\{T_{\alpha^{\prime}}^{\alpha}: \alpha^{\prime} \in A_{\alpha}\right\}$ for each $\alpha \in A$ are controlled semialgebraic $C^{2}$ tube systems for $\left\{C_{\alpha}: \alpha \in A\right\}$ and $\left\{B_{\alpha^{\prime}}^{\alpha}: \alpha^{\prime} \in A_{\alpha}\right\}$, respectively, and (1), (6)' and (7) are satisfied, where the definitions of $U_{J}$ etc. are not changed. We saw (8) under the conditions (6) and (7). But we can replace (6) by $(6)^{\prime}$ there. Hence (8) is now also satisfied.

Next we can assume $\left.\pi_{\alpha}\right|_{\operatorname{Int} B^{\alpha}}$ are of class $C^{\omega}$ as follows. First choose $\left\{\pi_{\alpha}\right\}$ so that Int $C_{\alpha}$ are $C^{\omega}$ manifolds, which is possible by the above arguments. Let $m \in \mathbb{Z}$. Inductively suppose $\left.\pi\right|_{\operatorname{Int} B^{\alpha}}$ are of class $C^{\omega}$ for $d_{\alpha}>m$, and let $\alpha_{0} \in A$ with $d_{\alpha_{0}}=m$. By the approximation theorem of $\left[\mathrm{S}_{1}\right],\left.\pi_{\alpha_{0}}\right|_{\operatorname{Int} B^{\alpha_{0}}}$ can be approximated by a semialgebraic $C^{\omega}$ map $\widehat{\pi}_{\alpha_{0}}$ : $\operatorname{Int} B^{\alpha_{0}} \rightarrow \operatorname{Int} C_{\alpha_{0}}$ in the 
$C^{2}$ topology. Then $\widehat{\pi}_{\alpha_{0}}$ is a diffeomorphism (see $\left[\mathrm{S}_{1}\right]$ ). Extend it to $\partial B^{\alpha_{0}}$ by setting $\widehat{\pi}_{\alpha_{0}}=\pi_{\alpha_{0}}$ there. Then $\widehat{\pi}_{\alpha_{0}}: B^{\alpha_{0}} \rightarrow C_{\alpha_{0}}$ is a homeomorphism by the definition of the $C^{0}$ topology, and $\left\{\pi_{\alpha}: \alpha \in A, \alpha \neq \alpha_{0}\right\} \cup\left\{\widehat{\pi}_{\alpha_{0}}\right\}$, $\left\{T_{\alpha}: \alpha \in A\right\}$ and $\left\{T_{\alpha^{\prime}}^{\alpha}: \alpha^{\prime} \in A_{\alpha}\right\}$ still satisfy (1), (6)' and (7) as shown below.

(1) is clear because we do not change $\left\{T_{\alpha^{\prime}}^{\alpha}\right\} ;(6)^{\prime}$ is also trivial if $C_{\alpha_{1}} \not \subset$ $\partial C_{\alpha_{0}}\left(\right.$ resp. $\left.B_{\alpha_{1}}^{\alpha} \not \subset \partial B_{\alpha_{0}}^{\alpha}\right)$.

Assume $C_{\alpha_{1}} \subset \partial C_{\alpha_{0}}$ in $(6)^{\prime}$, and that $(6)^{\prime}$ holds for $\varepsilon$. Let $\widehat{p}_{J}$, $\widehat{q}_{\alpha}$ and $\widehat{W}_{\alpha}(\varepsilon)$ be defined by $\left\{\pi_{\alpha}: \alpha \in A, \alpha \neq \alpha_{0}\right\} \cup\left\{\widehat{\pi}_{\alpha_{0}}\right\}$ in the same way as $p_{J}, q_{\alpha}$ and $W_{\alpha}(\varepsilon)$, and let $\left.\widehat{\varepsilon} \in\right] 0,1\left[\right.$ be so close to 0 that $\widehat{W}_{\alpha_{1}}(\widehat{\varepsilon}) \subset W_{\alpha_{1}}(\varepsilon)$. Then

$$
\left\{x \in \widehat{W}_{\alpha_{1}}(\widehat{\varepsilon}): q_{\alpha_{1}}(x) \neq \widehat{q}_{\alpha_{1}}(x)\right\} \subset W_{\alpha_{0}}(\widehat{\varepsilon})\left(=\widehat{W}_{\alpha_{0}}(\widehat{\varepsilon})\right) .
$$

Hence it suffices to see that the map

$$
\left.\left(q_{\alpha_{1}}-\widehat{q}_{\alpha_{1}}\right)\right|_{W_{\alpha_{0}}(\widehat{\varepsilon}) \cap \widehat{W}_{\alpha_{1}}(\widehat{\varepsilon})}: W_{\alpha_{0}}(\widehat{\varepsilon}) \cap \widehat{W}_{\alpha_{1}}(\widehat{\varepsilon}) \rightarrow \mathbb{R}^{n}
$$

is close to the zero map in the $C^{2}$ topology. By the definitions of $q_{\alpha_{1}}$ and $\widehat{q}_{\alpha_{1}}$ we have

$$
q_{\alpha_{1}} \circ q_{\alpha_{0}}=q_{\alpha_{1}}, \widehat{q}_{\alpha_{1}} \circ \widehat{q}_{\alpha_{0}}=\widehat{q}_{\alpha_{1}} \quad \text { on } W_{\alpha_{0}}(\widehat{\varepsilon}) \cap \widehat{W}_{\alpha_{1}}(\widehat{\varepsilon}), \quad \widehat{q}_{\alpha_{0}}=q_{\alpha_{0}} .
$$

Hence

$$
\left.\left(q_{\alpha_{1}}-\widehat{q}_{\alpha_{1}}\right)\right|_{W_{\alpha_{0}}(\widehat{\varepsilon}) \cap \widehat{W}_{\alpha_{1}}(\widehat{\varepsilon})}=\left.\left.\left(q_{\alpha_{1}}-\widehat{q}_{\alpha_{1}}\right)\right|_{\widehat{W}_{\alpha_{1}}(\widehat{\varepsilon}) \cap \operatorname{Int} C_{\alpha_{0}}} \circ q_{\alpha_{0}}\right|_{W_{\alpha_{0}}(\widehat{\varepsilon}) \cap \widehat{W}_{\alpha_{1}}(\widehat{\varepsilon})} .
$$

Therefore, we only need to see that

$$
\left.\left(q_{\alpha_{1}}-\widehat{q}_{\alpha_{1}}\right)\right|_{\widehat{W}_{\alpha_{1}}(\widehat{\varepsilon}) \cap \operatorname{Int} C_{\alpha_{0}}}: \widehat{W}_{\alpha_{1}}(\widehat{\varepsilon}) \cap \operatorname{Int} C_{\alpha_{0}} \rightarrow \mathbb{R}^{n}
$$

is close to the zero map in the $C^{2}$ topology since $q_{\alpha_{0}}$ is a proper semialgebraic $C^{2}$ retraction (for a proper semialgebraic $C^{2}$ map between semialgebraic $C^{2}$ manifolds $\varphi: M_{1} \rightarrow M_{2}$, the pull back by $\varphi$ : \{semialgebraic $C^{2}$ functions on $\left.M_{2}\right\} \rightarrow$ s semialgebraic $C^{2}$ functions on $\left.M_{1}\right\}$ is continuous). However, that is clear because

$$
\begin{aligned}
& \left.q_{\alpha_{1}}\right|_{\widehat{W}_{\alpha_{1}}(\widehat{\varepsilon}) \cap \operatorname{Int} C_{\alpha_{0}}}=\left.\pi_{\alpha_{0}} \circ q_{\alpha_{1}}^{\alpha_{0}} \circ \pi_{\alpha_{0}}^{-1}\right|_{\widehat{W}_{\alpha_{1}}(\widehat{\varepsilon}) \cap \operatorname{Int} C_{\alpha_{0}}}, \\
& \left.\widehat{q}_{\alpha_{1}}\right|_{\widehat{W}_{\alpha_{1}}(\widehat{\varepsilon}) \cap \operatorname{Int} C_{\alpha_{0}}}=\left.\pi_{\alpha_{0}} \circ q_{\alpha_{1}}^{\alpha_{0}} \circ \widehat{\pi}_{\alpha_{0}}^{-1}\right|_{\widehat{W}_{\alpha_{1}}(\widehat{\varepsilon}) \cap \operatorname{Int} C_{\alpha_{0}}},
\end{aligned}
$$

which holds by the definitions of $q_{\alpha_{1}}$ and $\widehat{q}_{\alpha_{1}}$, though $\pi_{\alpha_{0}}$ or $\widehat{\pi}_{\alpha_{0}}$ is not necessarily compatible with $\left\{T_{\alpha^{\prime}}^{\alpha}\right\}$ and $\left\{T_{\alpha^{\prime}}\right\}$.

In the same way, we see that also $q_{\alpha_{1}}^{\alpha}$ is a submersive $C^{2}$ retraction when $B_{\alpha_{1}}^{\alpha} \subset \partial B_{\alpha_{0}}^{\alpha}$. (Note that after replacing $\pi_{\alpha_{0}}$ by $\widehat{\pi}_{\alpha_{0}}$ we cannot preserve (6) nor compatibility of $\pi_{\alpha}$.) By the same reason (7) is kept. Therefore, we assume $\left.\pi_{\alpha}\right|_{\text {Int } B^{\alpha}}$ are all of class $C^{\omega}$.

Now we define a semialgebraic triangulation $(K, f)$ of $X$, as required in the theorem. Let the vertices of $K$ correspond to elements of $A$ and be denoted by $\left\{\sigma_{\alpha}: \alpha \in A\right\}$, and let vertices $\sigma_{\alpha_{1}}, \ldots, \sigma_{\alpha_{l}}$ span a simplex of $K$ 
if and only if $\left(\alpha_{\gamma(1)}, \ldots, \alpha_{\gamma(l)}\right) \in \mathcal{J}$ for some permutation $\gamma$ of $\{1, \ldots, l\}$. Then $K$ is a well defined simplicial complex called the dual complex of $\left\{C_{\alpha}\right\}$ (see $[\mathrm{R}-\mathrm{S}])$. For $J=\left(\alpha_{1}, \ldots, \alpha_{l}\right) \in \mathcal{J}$, let $\sigma_{J}$ denote the simplex spanned by $\sigma_{\alpha_{1}}, \ldots, \sigma_{\alpha_{l}}$. Thus $K=\left\{\sigma_{J}: J \in \mathcal{J}\right\}$. Let $m \in \mathbb{Z}$ and set

$$
\mathcal{J}_{m}=\left\{\left(\alpha_{1}, \ldots, \alpha_{l}\right) \in \mathcal{J}: d_{\alpha_{l}} \leq m\right\}, \quad K_{m}=\left\{\sigma_{J}: J \in \mathcal{J}_{m}\right\} .
$$

Note $K_{m}$ is a subcomplex of $K$. By induction assume we already have a semialgebraic triangulation $\left(K_{m}, f_{m}\right)$ of $\bigcup_{d_{\alpha} \leq m} C_{\alpha}$ such that $\left.f_{m}\right|_{\operatorname{Int} \sigma_{J}}$ is a $C^{\omega}$ diffeomorphism onto $U_{\pi_{\alpha_{1}}(0), J}$ for each $J=\left(\alpha_{1}, \ldots, \alpha_{l}\right) \in \mathcal{J}_{m}$. Then we need to extend $\left(K_{m}, f_{m}\right)$ to a semialgebraic triangulation $\left(K_{m+1}, f_{m+1}\right)$ of $\bigcup_{d_{\alpha} \leq m+1} C_{\alpha}$. For $J=\left(\alpha_{1}, \ldots, \alpha_{l}\right) \in \mathcal{J}_{m+1}-\mathcal{J}_{m}$, i.e., $d_{\alpha_{l}}=m+1$, define $\left.f_{m+1}\right|_{\sigma_{J}}: \sigma_{J} \rightarrow \mathrm{Cl} U_{\pi_{\alpha_{1}}(0), J}$ by $f_{m+1}\left(\sigma_{\alpha_{l}}\right)=\pi_{\alpha_{l}}(0)$ and

$$
\begin{array}{r}
f_{m+1}\left(t_{1} \sigma_{\alpha_{1}}+\cdots+t_{l} \sigma_{\alpha_{l}}\right) \\
=\pi_{\alpha_{l}}\left(\left(1-t_{l}\right) \pi_{\alpha_{l}}^{-1}\left(f_{m}\left(t_{1} \sigma_{\alpha_{1}} /\left(1-t_{l}\right)+\cdots+t_{l-1} \sigma_{\alpha_{l-1}} /\left(1-t_{l}\right)\right)\right)\right) \\
\quad \text { for } t_{1}, \ldots, t_{l} \in[0,1] \text { with } t_{1}+\cdots+t_{l}=1 \text { and } t_{l} \neq 1 .
\end{array}
$$

Then $\left(K_{m+1}, f_{m+1}\right)$ a semialgebraic triangulation of $\bigcup_{d_{\alpha} \leq m+1} C_{\alpha}$, and we obtain a semialgebraic triangulation $(K, f)$ as required.

It remains to prove that $\left\{U_{\pi_{\alpha_{1}}(0), J}: J=\left(\alpha_{1}, \ldots, \alpha_{l}\right) \in \mathcal{J}\right\}$ is a Whitney stratification. We show that, moreover,

$\left\{U_{\pi_{\alpha_{1}}(0), J}, U_{\pi_{\alpha_{1}^{\prime}}(0), J^{\prime}}\right\} \quad$ (resp. $\left.\left\{U_{\pi_{\alpha_{1}(0), J}}^{\alpha}, U_{\pi_{\alpha_{1}^{\prime}}(0), J^{\prime}}^{\alpha}\right\}\right)$ satisfies the Whitney condition for $J=\left(\alpha_{1}, \ldots, \alpha_{l}\right), J^{\prime}=\left(\alpha_{1}^{\prime}, \ldots, \alpha_{l^{\prime}}^{\prime}\right) \in \mathcal{J}$ and $\alpha \in A$ with $l>1, U_{\pi_{\alpha_{1}}(0), J} \subset \mathrm{Cl} U_{\pi_{\alpha_{1}^{\prime}}(0), J^{\prime}}-U_{\pi_{\alpha_{1}^{\prime}}(0), J^{\prime}}$ and $\alpha_{l}, \alpha_{l^{\prime}}^{\prime} \in A_{\alpha}$.

Here we also argue by induction. Let $m \in \mathbb{Z}$. Assume inductively (9) is proved for $d_{\alpha_{l^{\prime}}}<m$, and let $d_{\alpha_{l^{\prime}}^{\prime}}=m$. There are two possibilities $\alpha_{l}=\alpha_{l^{\prime}}^{\prime}$ or $\alpha_{l} \neq \alpha_{l^{\prime}}^{\prime}$.

Case of $\alpha_{l}=\alpha_{l^{\prime}}^{\prime}$. Set $J_{0}=\left(\alpha_{1}, \ldots, \alpha_{l-1}\right)$ and $J_{0}^{\prime}=\left(\alpha_{1}^{\prime}, \ldots, \alpha_{l^{\prime}-1}^{\prime}\right)$. Then $U_{\pi_{\alpha_{1}}(0), J_{0}} \subset \mathrm{Cl} U_{\pi_{\alpha_{1}^{\prime}}(0), J_{0}^{\prime}}-U_{\pi_{\alpha_{1}^{\prime}}(0), J_{0}^{\prime}}$ and $d_{\alpha_{l^{\prime}-1}^{\prime}}<m$. Hence $\left\{U_{\pi_{\alpha_{1}}(0), J_{0}}\right.$, $\left.U_{\pi_{\alpha_{1}^{\prime}}(0), J_{0}^{\prime}}\right\}$ (resp. $\left\{U_{\pi_{\alpha_{1}}(0), J_{0}}^{\alpha}, U_{\pi_{\alpha_{1}^{\prime}}(0), J_{0}^{\prime}}^{\alpha}\right\}$ ) satisfies the Whitney condition by hypothesis when $l-1>1$, and trivially when $l-1=1$. In particular, so does $\left\{U_{\pi_{\alpha_{1}}(0), J_{0}}^{\alpha_{l}}, U_{\pi_{\alpha_{1}^{\prime}}(0), J_{0}^{\prime}}^{\alpha_{l}}\right\}$. Then $\left\{U_{\pi_{\alpha_{1}}(0), J}^{\alpha_{l}}, U_{\pi_{\alpha_{1}^{\prime}}(0), J^{\prime}}^{\alpha_{l}}\right\}$ is a Whitney stratification by (1). Moreover, (9) holds since $\left.\pi_{\alpha_{l}}\right|_{\operatorname{Int} B^{\alpha_{l}}}: \operatorname{Int} B^{\alpha_{l}} \rightarrow \operatorname{Int} C_{\alpha_{l}}$ is a $C^{\omega}$ diffeomorphism and $U_{\pi_{\alpha_{1}}(0), J}^{\alpha_{l}}, U_{\pi_{\alpha_{1}^{\prime}}(0), J^{\prime}}^{\alpha_{l}} \subset \operatorname{Int} B^{\alpha_{l}}$.

Case of $\alpha_{l} \neq \alpha_{l^{\prime}}^{\prime}$. Set $J_{0}^{\prime}=\left(\alpha_{1}^{\prime}, \ldots, \alpha_{l^{\prime}-1}^{\prime}\right)$ as above. Then

$$
J=J_{0}^{\prime} \quad \text { or } \quad U_{\pi_{\alpha_{1}}(0), J} \subset \mathrm{Cl} U_{\pi_{\alpha_{1}^{\prime}}(0), J_{0}^{\prime}}-U_{\pi_{\alpha_{1}^{\prime}}(0), J_{0}^{\prime}},
$$

and hence $U_{\pi_{\alpha_{1}}(0), J}$ is equal to $U_{\pi_{\alpha_{1}^{\prime}}(0), J_{0}^{\prime}}$ or $\left\{U_{\pi_{\alpha_{1}}(0), J}, U_{\pi_{\alpha_{1}^{\prime}}(0), J^{\prime}}\right\}$ (resp. 
$\left.\left\{U_{\pi_{\alpha_{1}}(0), J}^{\alpha}, U_{\pi_{\alpha_{1}^{\prime}}(0), J^{\prime}}^{\alpha}\right\}\right)$ is a Whitney stratification. In both cases, (9) follows from (8). This completes the proof.

\section{References}

[G-al] C. G. Gibson et al., Topological Stability of Smooth Mappings, Lecture Notes in Math. 552, Springer, 1976.

[R-S] C. P. Rourke and B. J. Sanderson, Introduction to Piecewise-Linear Topology, Springer, 1972.

$\left[\mathrm{S}_{1}\right] \quad$ M. Shiota, Nash Manifolds, Lecture Notes in Math. 1269, Springer, 1987.

$\left[\mathrm{S}_{2}\right] \quad$ - Geometry of Subanalytic and Semialgebraic Sets, Progr. Math. 150, Birkhäuser, 1997.

Graduate School of Mathematics

Nagoya University

Chikusa, Nagoya, 464-8602

Japan

E-mail: shiota@math.nagoya-u.ac.jp 